Reprod. Nutr. Dévelop., 1987, 27 (2 B), 609-610.

\title{
GRF et sécrétion nocturne de GH chez l'enfant
}

Ch. SULTAN (1), M. RIALHE, J. TORREILles, Michèle LAPEYRONIE, P. GARANDEAU, R. JEAN

Service de Pédiatrie et Laboratoire de Biologie du Développement, CHU Montpellier, INSERM U.58, Montpellier.

Summary. Six children with severely retarded growth and normal growth hormone (GH) response to pharmacological tests were evaluated for their sleep-related $\mathrm{GH}$ secretion before and after the administration of growth-releasing factor (GRF). Our results suggest that GRF stimulates GH synthesis and spontaneous liberation during sleep. This preliminary study lends support to the potential therapeutic usefulness of GRF in these patients.

Introduction. L'exploration de la sécrétion de l'hormone de croissance (GH) chez l'enfant fait appel aux tests de stimulation pharmacologique et à l'analyse de la sécrétion physiologique de la GH pendant le sommeil (1). Chez certains enfants présentant un retard de croissance important, il apparaît une discordance entre une réponse normale de la $\mathrm{GH}$ aux tests dynamiques et une sécrétion nocturne de $\mathrm{GH}$ déficitaire. Dans ces cas, un dysfonctionnement neuro-sécrétoire (2) serait responsable d'une sécrétion nycthémérale de $\mathrm{GH}$ pathologique. Le but de ce travail a été de rechercher une action éventuelle du GRF (3-4) sur la synthèselibération de la GH pendant le sommeil, chez ces enfants.

Patients et méthodes. Cette étude porte sur 6 entants (âgés de 6 à 11 ans) présentant un retard statural supérieur à 3 déviations standard, avec valeurs normales de la GH obtenues à 2 tests de stimulation pharmacologique (hypoglycémie insulinique et glucagon-propanolol). Le protocole comporte l'évaluation de la sécrétion nocturne de la GH à $J-0$ et à $J+1$, après une injection de GRF $(2 \mu \mathrm{g} \mathrm{Kg}, \mathrm{IV}, \mathrm{GRF} 1-44$, Sanofi Recherche) à $8 \mathrm{~h}$ et à $16 \mathrm{~h}$ respectivement. $\mathrm{L}$ 'heure des repas est à $11 \mathrm{~h} 30$ et à $18 \mathrm{~h} 30$. Après mise en place d'un cathéter veineux, un prélèvement de sang est effectué toutes les 30 min après endormissement. La GH est dosée par méthode radio-immunologique, le nombre de prélèvements est constant pour chaque enfant qui est ainsi son propre témoin. La caractérisation de la sécrétion nocturne de GH comporte l'analyse du nombre de pics supérieurs à $7,5 \mathrm{ng} / \mathrm{ml}$, la concentration moyenne de $\mathrm{GH}$ et la surface sous la courbe.

Résultats et discussion. Les résultats de l'évaluation de la sécrétion de la $\mathrm{GH}$ nocturne à l'état de base $(J-0)$ et après GRF $(J+1)$ figurent sur le tableau. Si l'on admet que la concentration moyenne (intégrée) de la GH chez l'enfant normal est de l'ordre de $5 \mathrm{ng} / \mathrm{ml}$, il apparaît que les 6 patients étudiés présentent une hyposécrétion nocturne de $\mathrm{GH}$. Le nombre de pics de $\mathrm{GH}<$ à $7,5 \mathrm{ng} / \mathrm{ml}$ renforce cette notion. II est clair, par contre que l'administration de GRF là distance de la

(1) Tirés à part: Dr. Ch. Sultan, INSERM U.58, 60, rue de Navacelles, 34100 Montpellier. 
réponse dynamique en $\mathrm{GH}$ ) modifie le profil de sécrétion spontanée nocturne de l'hormone de croissance chez 5 des 6 enfants étudiés (patients 2-6).

TABLE 1

\begin{tabular}{lrrrrrrr}
\hline & \multicolumn{7}{c}{ Patients } \\
& & 1 & 2 & 3 & 4 & 5 & 6 \\
\hline \multirow{2}{*}{ Nombre de prélèvements } & $J-0$ & 19 & 18 & 17 & 14 & 12 & 16 \\
& $J+1$ & 19 & 18 & 17 & 14 & 12 & 16 \\
\hline \multirow{2}{*}{ Nombre de pics > 7,5 ng/ml } & $J-0$ & 3 & 1 & 3 & 0 & 2 & 2 \\
\hline \multirow{2}{*}{ Concentration moyenne de GH } & $J+1$ & 3 & 2 & 5 & 2 & 3 & 3 \\
\hline \multirow{2}{*}{ Surface sous la courbe } & $J-0$ & 2,9 & 2,1 & 3,8 & 2,0 & 4,3 & 3,7 \\
& $J+1$ & 3,7 & 6,3 & 9,5 & 10,7 & 9,4 & 6,4 \\
\hline
\end{tabular}

Ces données préliminaires sont en faveur d'une action du GRF sur la synthèse/libération de la $\mathrm{GH}$ nocturne. On peut donc admettre que dans ce cas, le retard de croissance relève d'une insuffisance de la sécrétion de $\mathrm{GH}$ pendant le sommeil ; aussi ces enfants pourraient théoriquement bénéficier d'un traitement par le GRF susceptible d'améliorer leur sécrétion nocturne de $\mathrm{GH}$ et par là même leur vitesse de croissance (5).

Remerciements. - Nous remercions de Dr. M. Olivier (Sanofi Recherche) pour I'attribution du GRF, M. Canazzi pour le dosage de la GH et C. Auge pour l'excellent travail de secrétariat.

(1) Sultan Ch., Amilhau D., Bonarde A., Garandeau P., Descomps B., Jean R., 1980. Ann. Pediat., 27, 145-151.

(2) Spiliotis B. E., August G. P., Hung W., Sonis W., Mendelson W., Bereu B. B., 1984. J. am. med. Assoc., 251, 2223-2230.

(3) Chatelain P., Bertrand J., David M., Sassolas G., Catbett N., François R., 1985. Arch. Fr. Pediatr., 42, 205-209.

(4) Guillemin R., Brazeau P., Bohlen P., Esch F., Ling N., Wehrenberg W. B., Bloch B., Mougin C., Zeitin F., Baird A., 1984. Rec. Prog. Horm. Res., 40, 233-299.

(5) Thorner M. O., Reschke J., Chitwood J., Rogol A. D., Rivier J., Vale W., Blizzard R. M., 1985. N. Engl. J. Med., 312, 4-9. 